\title{
The challenge of the asthma-COPD overlap syndrome (ACOS)
}

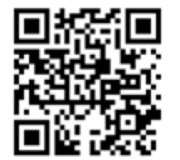

In the past, asthma airway inflammation was believed to be predominantly characterised by eosinophilic inflammation and type 2 helper $\mathrm{T}$ (Th2) lymphocytes, unlike chronic obstructive pulmonary disease (COPD), where inflammation is characterised predominantly by neutrophilic inflammation and CD8 lymphocytes. This difference in the pattern of inflammation makes the clinical extremes of asthma and COPD easily distinguishable, with differences in clinical picture and age of the patients. However, over the years, it has been observed that in older patients the presentation of asthma and COPD may converge clinically and the conditions may mimic each other. Airway remodelling that develops over time in some asthma patients leads to irreversible airway obstruction resembling COPD. In contrast, reversible airway obstruction can occur in patients with COPD, with the result that these patients may resemble those with asthma. The condition in which a person has clinical features of both asthma and COPD is called the asthma-COPD overlap syndrome (ACOS). The prevalence of ACOS is estimated to be $15-45 \%$ in people with obstructive airway disease and increases with age.

In a recent review, Postma and Rabe ${ }^{[1]}$ discuss studies that have shown the heterogeneity of pathophysiology of asthma and COPD. Therefore it may be difficult to distinguish asthma from COPD in patients who have pathophysiological and clinical features of both. The authors emphasised that there is still a paucity of data on how to diagnose and treat ACOS and answers to the primary review questions (i.e. how would one make appropriate ACOS diagnosis and what is the appropriate treatment for ACOS?) are not evidencebased. Nevertheless, the review highlighted important points on pathophysiology, clinical features, diagnosis and treatment of obstructive airway disease assuming that asthma and COPD are two extreme ends of the disease spectrum with ACOS in between.

\section{Pathophysiology of asthma, COPD and ACOS and clinical implications}

Studies have shown substantial heterogeneity in progressive airway obstruction, bronchial hyper-responsiveness, reversibility of airway obstruction, atopy, airway inflammation and exhaled nitric oxide (FeNO) among patients with asthma or COPD.

From early adulthood, forced expiratory volume in 1 second $\left(\mathrm{FEV}_{1}\right)$ normally declines by $\sim 25-50 \mathrm{ml}$ annually. The decrease is greater in obstructive airway disease. Although the decrease is more in COPD than asthma, there is no convincing evidence that the rate of $\mathrm{FEV}_{1}$ decline can be used to distinguish between asthma and COPD.

The global trend of increasing life expectancy shifts the median age of the population with asthma upward. This increases the probability of overlap with COPD as defined by $\mathrm{FEV}_{1}$; hence, the estimated prevalence of ACOS is highly age-dependent.

Bronchial hyper-responsiveness, which was initially thought to be a hallmark of asthma and a marker of eosinophilic inflammation, is also present in some COPD patients. It is driven by multiple factors (allergen and non-allergen) and may not be as responsive to steroids as it is in asthma.
Reversibility of airway obstruction after inhalation of a bronchodilator drug can diminish or even disappear with longstanding asthma and treatment with a bronchodilator drug or inhaled steroids. Therefore, lack of full reversibility does not rule out an asthma diagnosis. Reversibility of airway obstruction is frequently present in COPD as well; reversibility was observed in up to $50 \%$ of patients with COPD.

Atopy, which is a risk factor for asthma, has also been found to be a risk factor for COPD in two studies (ECLIPSE and EUROSCOP). COPD patients with atopy are somewhat younger, more likely male, with higher body mass index and more likely to benefit from steroid treatment.

There is evidence from bronchial biopsy/sputum studies/exhaled breath studies that there is substantial heterogeneity in mucosal inflammation. Asthma patients with severe or late-onset disease or chronic infections or who smoke, may also exhibit neutrophilic inflammation and CD8 cells in the airways, both of which were once believed to be hallmarks of COPD. Therefore, the absence of eosinophilia and the lack of a response to inhaled glucocorticoids in a patient does not rule out asthma. Similarly, some COPD patients may have eosinophilic inflammation which is not usually steroid responsive.

Exhaled FeNO levels are lower in smokers than in non-smokers, which makes measurements of FeNO levels less useful for differentiating asthma from COPD.

\section{Diagnosis of ACOS}

So far there is no agreed specific ACOS diagnosis definition to guide clinicians. However, depending on patient age, smoking history, atopy history, bronchial hyper-responsiveness, airway obstruction reversibility and symptoms, patients with obstructive airway disease can either have 'easy' asthma and 'easy' COPD or overlap syndrome (ACOS). ACOS patients can evolve either from asthma or from COPD.

The Global Initiative for Asthma (GINA) and Global Initiative for Chronic Obstructive Lung Disease (GOLD) propose that if three or more features of either asthma or COPD are present, then that diagnosis is suggested; if there are similar numbers of features of asthma and COPD, the diagnosis of ACOS should be considered.

- 'Easy asthma' patients: characteristically young (about 21 years), with a history of atopy, non-smokers, recurrent wheezing/ dyspnoea, reversible airway obstruction and bronchial hyperresponsiveness.

- 'Easy COPD' patients: characteristically older (about 65 years), with no atopy history, heavy smokers (about 95 pack-years), chronic dyspnoea, no wheezing/reversible airway obstruction/ bronchial hyper-responsiveness.

- ACOS stemming from asthma: middle-aged (about 45 years) with atopy history, non-smoker, chronic dyspnoea with flares and wheezing, bronchial hyper-responsiveness, but no airway obstruction reversibility.

- ACOS stemming from COPD: middle-aged (about 45 years) with atopy, smoker (20 pack-years), chronic dyspnoea with flares, wheezing, reversible airway obstruction and with or without bronchial hyper-responsiveness. 


\section{Clinical relevance of ACOS and treatment for ACOS}

Despite GINA and GOLD recognising ACOS as a clinical reality, there is still need for more evidence on clinical phenotypes and underlying mechanisms that will help in devising a specific definition of ACOS and its treatment.

There is the possible danger of overdiagnosing ACOS, thereby overtreating COPD patients with inhaled glucocorticoids, if different definitions of ACOS are used.

Inconsistent definitions used in treatment studies make it almost impossible to determine the most effective therapy for an individual ACOS patient.

For 'easy' asthma and 'easy COPD', clear step-wise treatment approaches have been provided by GINA and GOLD, respectively. These approaches also include treatment for exacerbation reduction and comorbidities.

- 'Easy asthma': Inhaled glucocorticoids in combination with bronchodilator drugs (short- and long-acting beta-agonists (LABAs) are the main pillars. Leukotriene-receptor antagonists are an alternative choice in milder disease cases. For severe allergic asthma with appropriate IgE levels, anti-IgE treatment is an approved option.

- 'Easy COPD': The main emphasis is on smoking cessation and the use of LABAs and long-acting muscarinic antagonists (LAMAs). The role of inhaled glucocorticoids is still debatable; however, it is accepted for patients with more severe disease and those with frequent exacerbations.

- ACOS stemming from asthma: There are no firm treatment guidelines. However, due to lack of randomised clinical trials, treatment with inhaled glucocorticoids should be continued in patients with long-standing asthma, even if a component of irreversible airway obstruction develops; leukotriene modifiers may be of value in those with atopy. Combination therapy with a LAMA and a LABA is a reasonable approach for patients with more severe asthma or COPD or with overlapping conditions.

- ACOS stemming from COPD: Because of the current evidence that some COPD patients may have reversibility, eosinophilia and bronchial hyper-responsiveness, such patients may benefit from inhaled glucocorticoids.

\section{Conclusion}

With no clinical trials on ACOS, the authors believe it is still premature to recommend the designation of ACOS as a disease entity. There is need for further research to obtain a standardised definition of ACOS and its treatment; unique biomarkers will settle the debate.

\section{Ndaziona P K Banda}

Division of Pulmonology, Chris Hani Baragwanath Academic Hospital and Faculty of Health Sciences, University of the Witwatersrand, Johannesburg, South Africa

\section{References}

1. Postma DS, Rabe KF. The asthma-COPD overlap syndrome. N Engl J Med 2015;373(13):1241-1249. [http://dx.doi.org/10.1056/NEJMra1411863]

S Afr Respir J 2015;21(4):110-111. DOI:10.7196/SARJ.2015.v21i4.49

\section{High-flow oxygen therapy in acute hypoxaemic respiratory failure}

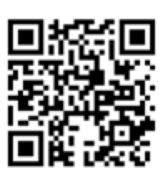

High-flow oxygen therapy allows for the delivery of heated and humidified oxygen via nasal cannula at high flow rates, thereby generating low levels of positive pressure in the upper airways. Additionally, this technique allows for the titration of the fraction of inspired oxygen as well. ${ }^{[1-3]}$ High-flow oxygen therapy has been shown to result in better comfort and oxygenation in patients with acute respiratory failure in previous studies. However, evidence for high-flow oxygen therapy on intubation rates and mortality is lacking.

Frat et al.$^{[4]}$ in a recent trial compared intubation rates within 28 days in patients who were assigned to either high-flow oxygen therapy, standard facemask oxygen therapy or non-invasive positive pressure ventilation. Secondary outcomes were the number of ventilator-free days at day 28 and all-cause mortality at 90 days. In this multicentre, open-label trial 310 patients across 23 intensive care units in Belgium and France were randomly assigned to each group. All patients included in the study had acute hypoxaemic respiratory failure without hypercapnia, and a partial pressure to fraction of respired oxygen ratio of $<300 \mathrm{~mm} \mathrm{Hg}$.

\section{Results}

The intubation rate was non-significantly different in the three groups: $38 \%$ in the high-flow oxygen group, $47 \%$ in the standard facemask oxygen group, and $50 \%$ in the non-invasive ventilation group $(p=0.18)$. The number of ventilator-free days at day 28 was analysed as a secondary outcome, and was significantly lower in the high-flow oxygen group (24 (8) days compared with 22 (10) in the facemask group and 19 (12) in the non-invasive ventilation group); for all analyses $p=0.02$. High-flow oxygen therapy was also found to significantly lower 90-day mortality as a secondary outcome compared with standard oxygen therapy and non-invasive ventilation. The hazard ratio for death at 90 days was 2.01 (95\% confidence interval (CI) 1.01 - 3.99) when comparing standard oxygen with high-flow oxygen therapy $(p=0.046)$ and $2.5(95 \% \mathrm{CI}$ 1.31 - 4.78) with non-invasive ventilation compared with high-flow oxygen ( $p=0.006$ ). Given the findings of this study, high-flow oxygen therapy appears to be a reasonable alternative to facemask oxygen and non-invasive ventilation in patients with acute hypoxaemic respiratory failure and may confer a survival benefit.

\section{Laila Suleman}

Department of Pulmonology and Critical Care, Charlotte Maxeke Johannesburg Academic Hospital and University of the Witwatersrand, Johannesburg, South Africa

\section{References}

1. Chanques G, Riboulet F, Molinari N, et al. Comparison of three high flow oxygen therapy devices: A clinical physiological cross-over study. Minerva Anestesiol 2013;79(12):1344-1355.

2. Corley A, Caruana LR, Barnett AG, Tronstad O, Fraser JF. Oxygen delivery through high-flow nasal cannulae increase end-expiratory lung volume and reduce respiratory rate in post-cardiac surgical patients. Br J Anaesth 2011;107(6):998-1004. [http:// dx.doi.org/10.1093/bja/aer265]

3. Parke RL, Eccleston ML, McGuinness SP. The effects of flow on airway pressure during nasal high-flow oxygen therapy. Respir Care 2011;56(8):1151-1155. [http://dx.doi. org/10.4187/respcare.01106]

4. Frat JP, Thille AW, Mercat A, et al. High-flow oxygen through nasal cannula in acute hypoxaemic respiratory failure. N Engl J Med 2015;372(23):2185-2196. [http://dx.doi. org/10.1056/NEJMoa1503326]

S Afr Respir J 2015;21(4):111. DOI:10.7196/SARJ.2015.v21i4.48 